\title{
Clinicopathologic Profile and Outcome of Extranodal Diffuse Large B-Cell NHL: Egyptian National Cancer Institute Experience
}

\author{
Ola Khorshed; M. D. ${ }^{1}$, AmanyHelal; M.D. ${ }^{1}$, Yasser A. Sallam; M. D', \\ AsmaaSalama; M.D. ${ }^{2}$ Mohammad Amin; Msc. ${ }^{1}$ \\ ${ }^{1}$ Medical Oncology Department, National Cancer Institute, \\ Cairo University,Egypt \\ ${ }^{2}$ Pathology Department, National Cancer Institute, \\ Cairo University, Egypt
}

Received 11 March 2015; Accepted 6 July 2015

\begin{abstract}
Background: Primary extranodal diffuse large B-Cell lymphoma (PE-DLBCL) accounts for about one-third of all cases of DLBCL. We reviewed the clinical and pathological characteristics of cases with PE-DLBCL presented to NCI, Egypt.

Patients and methods: We retrospectively studied patients with pathologically documented PE-DLBCL presented to the National Cancer Institute between January 2008 and December 2010. Revision of histopathology and sub-classification of patients to germinal centre (GC) DLBCL and non-GC-DLBCL through immunostaining of CD10, BCL-6 and MUM-1 were done. Cases that were CD10+/BCL-6 \pm / MUM-1- were categorised as GC-DLBCL, while cases that were CD10-/BCL-6 $\pm / M U M-1+$ were categorised as non-GC-DLBCL. Clinical data regarding baseline characteristics, chemotherapy given and the response to chemotherapy was collected.

Results: A total of 57 patients of PE-DLBCL were included in the study. Mean age was 48.9 years (range 21-78), and 32 patients (56.1\%) were females. Most frequent locations were gastrointestinal and liver (29.9\% and $22.9 \%$; respectively). Forty-two patients $(73.7 \%)$ were sub-classified as non-GC-DLBCL. The patients who were stage III/IV were more common (52.6\%). Fifty-two patients received CHOP, 5 received CVP, and 6 patients received radiotherapy after finishing chemotherapy. Complete response rate (CR) was $64.9 \%$, there was no difference in the CR $(66.7 \%, 64.3 \%)$ and Overall Survival $(53.3 \%, 52.4 \%)$ in GC-DLBCL group in comparison to non-GC-DLBCL group $(p=0.24$ and 0.6 ; respectively). The CR and OS for patients with low international prognostic index (IPI) were significantly better than patients with intermediate or high IPI ( $p=0.008$ and 0.08 , respectively).

Conclusion: Non-GC-DLBCL is more common than GC-DLBCL in PE-DLBCL in Egyptian population. The most affected sites are gastrointestinal and liver reflecting the association between high frequency of hepatitis B and $C$ and hepatic non-Hodgkin's lymphoma (NHL). Stage of the disease and IPI remains the most important prognostic factor for PE-DLBCL.
\end{abstract}

Keywords: Extranodal $\bullet N H L \cdot$ clinicopathologic profile

(c) De Gruyter Open

\section{Introduction}

Approximately one-third of the non-Hodgkin's lymphoma $(\mathrm{NHL})$ arise in tissues other than the lymph nodes, hence, they are usually termed extranodal lymphomas (1). Diffuse large B-cell Lymphoma (DLBCL) is the most common extranodal lymphoma. It has been shown that during the last two decades, the incidence of lymphomas has increased and that extranodal lymphomas increased more rapidly than nodal (2). There are great differences in the incidence of extranodal lymphomas (out of all $\mathrm{NHL}$ ) among countries; USA $24 \%$, Canada $27 \%$, Israel
$36 \%$, Lebanon $44 \%$, Denmark $37 \%$, Netherlands $41 \%$, Italy $48 \%$ and Hong Kong 29\% (3). In Egypt, extranodal lymphoma was reported in $23 \%$ of $\mathrm{NHL}$ cases (4).

Although considered a single entity in the WHO classification, DLBCL most likely includes different clinicopathologic entities with different morphology and immunophenotyping. In 2004, Hans and colleagues proposed an immunohistochemical method to classify patients with DLBCL into germinal centre (GC) and nonGC (5). They demonstrated that the non-GC subtype was associated with a worse prognosis compared to the GC subtype in patients treated with chemotherapy. 
This subclassification can be performed by panel of monoclonal antibodies against the following antigens: CD10, MUM1, BCL-6 and consequently, patients are clustered into two groups according to their immunophenotypic profile in order to assess the pattern of differentiation.

The main purpose of this study was to identify the clinical features and outcome of extranodal DLBCL among Egyptian patients, as well as their classification according to Hans' system.

\section{Patients and methods}

We studied retrospectively all patients diagnosed as extranodal DLBCL presented to the Medical Oncology Clinic, National Cancer Institute, Cairo University during the period from January 2008 to December 2010. Fiftyseven patients had available paraffin blocks and clinical data and consequently, were included in the study. Clinical data were retrieved from patients' files including; age, sex, site, performance status, laboratory investigations (CBC, liver and kidney functions, bone marrow aspirate and biopsy), radiological data (CT neck, chest, abdomen and pelvis), upper and lower Gastrointestinal Track endoscopy for GIT lymphoma, stage according to modified Ann Arbor criteria, initial chemotherapy, response, treatment of refractory/relapsed cases, date of relapse, date of last visit and state.

The paraffin blocks of cases were retrieved and Hx\&E sections were prepared for histopathologic evaluation in correlation with the immunostained slides for revision of their immunophenotyping. Three sections were prepared from each paraffin block (4 microns thick) and immunostained using primary monoclonal antibodies against CD10 (clone 56C6, ready to use, DAKO), BCL-6 (clone PG-B6p, ready to use, DAKO), and MUM-1 (clone MRQ-8, ready to use, Cell Marque) using Bench Mark VentanaAutostainer (XT). Positive reaction was considered at cut-off point $30 \%$ of all markers. Cases were classified according to Hans Algorithm4 into; 1. Positive CD10 meant that it was of GC subtype and it was must for all cases having nonGC tumours to have negative CD10 immunostaining, 2. Cases that had negative CD10 with negative BCL-6 were all of non-GC group, while cases with negative CD10 and positive BCL-6 were further evaluated for MUM-1; if positive MUM-1 then they were GC subtype but if negative, they were non-GC.

Disease-free survival was calculated from the date of complete remission till the date of relapse/death or last follow-up for non-relapsed patients. Overall survival was calculated from the date of diagnosis to the date of death/last follow-up.

Data were analysed using SPSS (version 16). Numerical data were expressed as means with standard deviation and range. Qualitative data were expressed as frequency and percentage. Survival analysis was done using the Kaplan-Meier method. Comparison between survival curves was done using the Log-rank test. P value $<0.05$ was considered significant.

\section{Result}

The patients included 25 men (44\%) and 32 women $(56 \%)$ with a median age of 48 years (range $21-78$ years). Tumour sites included; gastrointestinal (17 cases, $29.9 \%$ ), liver (13 cases, $22.9 \%$ ), upper aerodigestive (8 cases, $14.1 \%$ ), bone (5 cases, $8.8 \%$ ), lung and pleura ( 4 cases, $7 \%$ ), kidney ( 2 cases, $3.5 \%$ ), spleen ( 2 cases, $3.5 \%$ ), soft tissue (2 cases, $3.5 \%$ ), testis ( 1 case, $1.7 \%$ ), ovary ( 1 case, $1.7 \%$ ), breast ( 1 case, $1.7 \%$ ) and parotid (1 case, $1.7 \%$ ). About $47.4 \%$ of cases presented by localised disease (stage I or II) while $54.6 \%$ had advanced stage initially (stage III or IV). Of 57 patients, $15(26.3 \%)$ were considered of GC subtype and 42 $(73.7 \%)$ were considered of non-GC subtype. Out of 15 patients of GC subtype; 6 patients (40\%) presented with localised disease while 9 patients (60\%) presented with advanced disease. On the other hand, in the non-GC subtype; localised disease and advanced disease were equally distributed (50\% each). Regarding IPI, patients with GC subtype were equally distributed among low, intermediate and high-risk categories (5patients, 33.3\% each), but patients with non-GC subtype were more common at low risk than intermediate and high risk (45.2\%, 33.3\% and 21.4\%; respectively).

Fifty-two patients received $\mathrm{CHOP}$ as the firstline chemotherapy, at that time, patients could not receive Rituximab, which is standard for a $\mathrm{B}$-cell $\mathrm{NHL}$, as Rituximab was not avialable for our patients due to lack of resources and 6 of them received Involved Field Radiation Therapy (IFRTH) after the end of 4-6 cycles. Five cases received COP because of old age (> 60 years, 4 cases), poor performance status (2 cases) and/or associated cardiac disease (one case). Six patients received radiotherapy after finishing chemotherapy. Thirty-seven patients (64.9\%) achieved CR while eighteen patients (31.6\%) failed. No significant difference between GC and non-GC groups regarding frequency of $\mathrm{CR}(66.7 \%$ versus $64.3 \%$; respectively, $\mathrm{p}=$ $0.247)$. On the contrary, stage had marked impact on the incidence of CR; as it occurred in $85.2 \%$ in patients with 


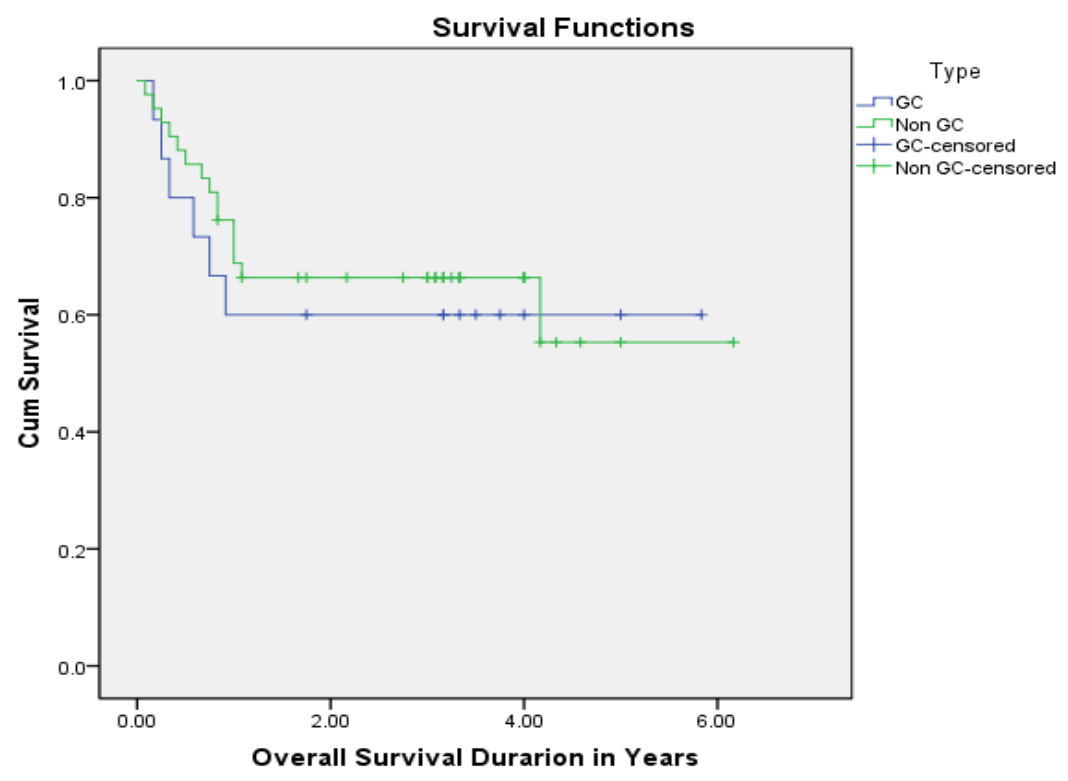

Figure 1. Relation between Overall Survival and DLBCL subtyping into GC and non-GC. DLBCL: diffuse large B-Cell lymphoma; GC: germinal centre.

localised disease comparison to $53.3 \%$ in patients with advanced disease, with statistical significant difference $(p=0.008)$. Relapse occurred in seven patients $(18.9 \%)$ out of all patients who achieved CR. It occurred either localised in the same primary site or disseminated in CNS, skin, liver and kidney.

The 3-year OS survival for the entire patients was $54.3 \%$. The main causes of death were neutropenia, disease progression and liver cell failure. No difference was found between GC subtype and non-GC subtype regarding OS (figure 1), 53.3 versus $52.4 \%$ ( $p=0.656$ ). Advanced stage had worsened the OS by $40 \%$ in comparison to localised disease where OS was $66.7 \%$ (figure 2), but statistical significant difference could not be achieved $(p=0.19)$. OS for patients with low-risk IPI was significantly better than patients with intermediate or high IPI $(p=0.08)$.

\section{Discussion}

$\mathrm{NHL}$ is a heterogeneous neoplasm regarding presentation, response to therapy and outcome. The classification of NHL as primary nodal or extranodal is a controversial issue (6). Patients with purely nodal or extranodal are easily classified. In some studies, only localised extranodal lymphomas had been defined as PE NHL (7). In other studies, classification of NHL is mainly based on the clinicopathologic features, but not taking into consideration the primary site of $\mathrm{NHL}$ (8).
Currently, the prognosis for patients with DLBCL is estimated by using the clinical parameters of the IPI that reflects mixture of underlying biologic and genetic differences. Some studies tried to elucidate the underlying factors using molecular techniques using cDNA microarray dividing DLBCL into GC B-cell like, activated $B$-cell like (ABC) and type 3 gene expression profile (9). The latter is heterogeneous and has a poor outcome similar to the ABC group, both lumped into nonGC group (10). Because this technology is expensive and not generally available, a simpler, more widely available method to subclassify DLBCL into molecularly distinct and prognostically significant groups; GC and non-GC, using immunohistochemistry would have wide applicability. The most commonly used panel is CD10, BCL-6, and MUM1. However, the results are conflicting, some authors found no difference in the outcome between $G C$ and non-GC groups $(11,12)$ while others obtained better survival for GC group $(10,13)$.

In our study, 57 cases were included with primary tumour sites included; gastrointestinal (17 cases, $29.9 \%$ ), liver (13 cases, $22.9 \%$ ), upper aerodigestive (8 cases, $14.1 \%$ ), bone (5 cases, $8.8 \%$ ), lung and pleura ( 4 cases, $7 \%$ ), kidney ( 2 cases, $3.5 \%$ ), spleen ( 2 cases), soft tissue ( 2 cases), testis ( 1 case, $1.7 \%$ ), ovary (1 case), breast (1 case) and parotid (1 case). We noticed high prevalence of liver being affected than Western as shown by Lopez-Guillermo (14), who documented liver cases in only $2 \%$ of their patients. This could be due to the high prevalence of hepatitis $B$ and $C$ in our country 


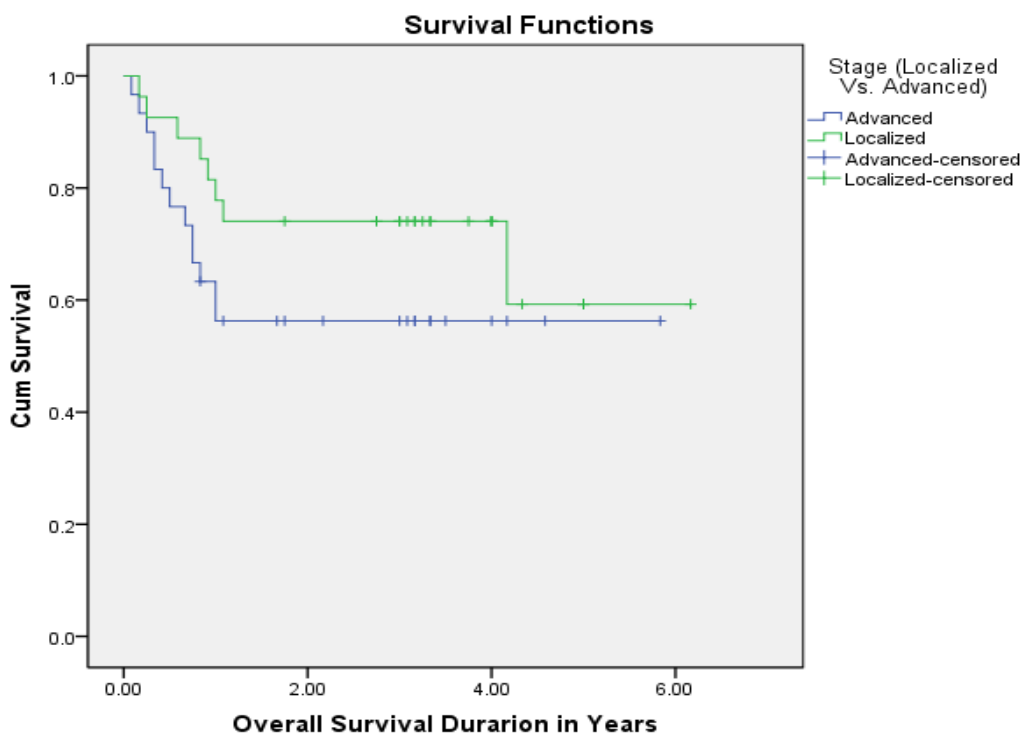

Figure 2: Relation between Overall Survival and stage (localised versus advanced).

predisposing to increase in liver $\mathrm{NHL}$ (15). On the other hand, their CNS cases are about $2.5 \%$ comparable to none in our study.

In our study, 37 patients (64.9\%) achieved complete remission (CR), which is lower than what was reported by Lal et al. (2008) 16, which was $74.4 \%$, but the difference could be attributed to that all their cases were stage I and II, while $54.6 \%$ of our cases were advanced stage (III and IV). No significant difference between GC and non-GC groups regarding frequency of CR (66.7 versus $64.3 \%, p=0.247)$. On the contrary, stage had marked impact on the incidence of CR; as it occurred in $85.2 \%$ in patients with localised disease in comparison with $53.3 \%$ in patients with advanced disease, with statistical significant difference $(p=0.008)$. This was coincident with what was found by Lopez-Guillermo (14) who reported high $\mathrm{CR}$ among patients of early stage but not correlated with typing into GC or non-GC.

\section{References}

[1] Harris NL (2001) Mature B-cell neoplasms In: WHO Classification of Tumors: Pathology and Genetics of Tumors of Hematopoietic and Lymphoid Tissues, Jaffe EJ, Harris NL, Stein $\mathrm{H}$ et al. (eds), Lyon, France, IARC Press, p 121- 126

[2] Jemal A, Tiwari RC, Murray T, Ghafoor A, Samuels A, Ward E, et al. Cancer statistics, 2004. CA Cancer $\mathrm{J}$ Clin 2004;54:8-29

[3] Groves FD, Linet MS, Travis LB, et al (2000) Cancer surveillance series: Non-Hodgkin's lymphoma
The 3-year OS survival for the entire patients was $54.3 \%$ with no difference was found between GC subtype and non-GC subtype regarding OS, 53.3 versus $52.4 \%$ ( $p=0.656$ ). Advanced stage and intermediate/ high-risk IPI were unfavourable prognostic factor of OS. Same findings were reported in other studies (14) where 5 -year OS was $52 \%$ with unfavourable predictors were poor IPI and advanced stage.

In conclusion, extranodal DLBCL showed a high prevalence of liver in Egyptian population, probably due to widespread hepatitis B and C virus in Egypt. Stage and IPI are the main prognostic factors for patients' outcome. Classification of DLBCL into GC and nonGC subtypes remains a controversial issue with no consensus regarding its relation to prognosis, requiring more extensive studies on larger population.

incidence by histologic subtype in the United States from 1978 through 1995. J Natl Cancer Inst 92:1240-1251.

[4] Mokhtar N, Gouda I, and Adel I (2007) Lymphoma in: Cancer Registry 2003-2004, p 36.

[5] Hans CP, Weisenburger DD, Greiner TC, et al. (2004) Confirmation of the molecular classification of diffuse large B-cell lymphoma by immunohistochemistry using a tissue microarray. Blood; 103:275-282. 
[6] Krol ADG, leCessie S, and Snijder S (2003) Primary extranodal non-Hodgkin's lymphoma (NHL): The impact of alternative definitions tested in the Comprehensive Cancer Center West populationbased NHL registry. Ann Oncol; 14; 131-9.

[7] Lossos IS, Czerwinsli DK, Alizadeh AA et al. (2004) Prediction of survival in diffuse large B-cell lymphoma based on the expression of six genes. $\mathrm{N}$ Engl J Med; 350:1828-37

[8] Pileri SA, Dimhofer S, Went Ph, et al. (2002) Diffuse large B-cell lymphoma: One or more entities? Present controversies and possible tools for its subclassification. Histopathology; 41:482-509.

[9] Rosenwald A, Wright G, Chan WC, et al (2002) The use of molecular profiling to predict survival after chemotherapy for diffuse large B-cell lymphoma. N Engl J Med; 346:1937-1947.

[10] McClintock S, Perkins SL, Cleveland RP, et al. (2003) Immunohistochemical expression pattern of germinal center and activation B-cell markers correlates with prognosis in diffuse large B-cell lymphoma. Mod Pathol; 16:244.

[11] Colomo L, Lopez-Guillermo A, Perales M, et al. (2003) Clinical impact of the differentiation profile assessed by immunophenotyping in patients with diffuse large B-cell lymphoma. Blood; 101:78-84.
[12] Linderoth J, Jerkeman M, Cavallin-Stahl E, Kvaloy S, Torlakovic E. (2003) Immunohistochemical expression of CD23 and CD40 may identify prognosticallyfavorable subgroups of diffuse large B-cell lymphoma: a Nordic Lymphoma Group Study. Clin Cancer Res; 9:722-728.

[13] Barrans SL, Carter I, Owen RG, et al. Germinal center phenotype and bcl-2 expression combined with the International Prognostic Index improves patient risk stratification in diffuse large B-cell lymphoma. Blood; 99:1136-1143.

[14] Lopez-Guillermo A, Colomo L, Jimenez M, et al. (2005) Diffuse large B-cell lymphoma: clinical and biological characterization and outcome according to the nodal or extranodal primary origin. Journal of Clinical Oncology, 23; 2797.

[15] Negri E, Little D, Boiocchi M, et al. (2004) B-cell non-Hodgkin's lymphoma and hepatitis $C$ virus infection: A systematic review. International Journal of Cancer; 111:1-8.

[16] Lal A, Bhurgri Y, Vaziri I, Rizvi NB, et al. (2008) Extranodal Non-Hodgkin's Lymphomas - A retrospective review of clinicopathologic features and outcomes in comparison with nodal nonHodgkin lymphomas. Asian Pacific Journal of Cancer Prevention; 9:453. 